\title{
TELEVISIÓN EN COLOMBIA: \\ Surgimiento de los canales regionales
}

TV IN COLOMBIA:

Emergence of regional channels

Ana Patricia García Ángel*

\section{Resumen}

El artículo expone que la televisión en Colombia surge unida al poder, centralizada y como un medio de comunicación cuyos propósitos educativos y culturales se quedaron en el papel. Expone algunos de los acontecimientos que rodearon el montaje de la televisión Nacional. Contextualiza la aparición de los canales regionales: Teleantioquia, Telecaribe, Telepacífico, Telecafé, Canal Capital, Tvandina (Hoy Canal 13), Televisión Regional del OrienteTRO y Teleislas.

El texto hace parte del marco referencial de la tesis "La televisión regional en Colombia" presentada por la autora en el marco de sus estudios de Magister en Comunicación en el Espacio Visual Iberoamericano en la Universidad Internacional de Andalucía-España.

Palabras Clave: Historia de la Televisión en Colombia, Canales Regionales, Teleantioquia, Telecaribe, Telepacífico, Telecafé, Canal Capital, Tvandina, Televisión Regional del Oriente- TRO, Teleislas.

\section{Sumary}

The article present that the television was born in Colombia linked to power, originated from the center of the country and as a communication medium with educational and cultural purposes raised in the paper. Exposes some of the events surrounding the creation of the National Television. Contextualizes the appearance of regional channels: Teleantioquia, Telecaribe, Telepacífico, Telecafé, Canal Capital, Tvandina (Today Canal 13), Televisión Regional del Oriente- TRO and Teleislas.

The text is part of the framework of the thesis "Regional television in Colombia,"presented by the author in the context of studying Masters in Visual Communication at the Ibero -American Space at the International University of Andalusia- España.

Keywords: Television History in Colombia, Regional Channels, Teleantioquia, Telecaribe, Telepacífico, Telecafé, Canal Capital, Tvandina, Televisión Regional del Oriente- TRO, Teleislas.

\footnotetext{
* Comunicadora Social-Periodista de la Universidad de Antioquia, Especialista en Televisión de la Universidad Pontificia Bolivariana. Magister en Comunicación en el Espacio Visual Iberoamericano de la Universidad Internacional de AndalucíaEspaña. Gerente de programación por más de 25 años en la industria de la televisión. Docente de cátedra del Politécnico Jaime Isaza Cadavid y otras universidades de Medellín.
} 


\section{Introducción}

$\mathrm{E}$ 1 surgimiento de la televisión en Colombia tiene lugar el 13 de junio de 1954; un año después de que el General Gustavo Rojas Pinilla, destituyera mediante un golpe militar al presidente conservador Laureano Gómez.

El anuncio del servicio de televisión en Colombia lo hace la Oficina de Información y Prensa del Estado -ODIPE-, una especie de Ministerio de Información propuesto por Rojas Pinilla en sus primeros actos de gobierno, en donde nombra como director al antioqueño Jorge Luis Arango, experto en divulgación, con trayectoria en el Departamento de Extensión Cultural del Ministerio de Educación.

La noticia de la naciente Cadena Nacional de Televisión se publica en la edición del mes de marzo de 1954 en el boletín de programas de la Radiodifusora Nacional de Colombia.

\section{TV oficial y centralista}

En el mes de octubre el General Rojas Pinilla, ante la lentitud mostrada por el grupo de Ministros designados para dar marcha al proceso de montaje de la televisión, le encomendó a Arango iniciar dicho poryecto, a través de la Oficina de Información y Prensa del Estado -ODIPE- y particularmente a través de la Radiodifusora Nacional. Jorge Luis Arango, designa a Fernando Gómez Agudelo como director de la Radiodifusora Nacional. En aquella época Gómez era estudiante de derecho, melómano, aficionado a los estudios de reproducción electrónica del sonido y columnista de música en el periódico conservador El Siglo.

De esta manera, bajo dependencia directa de la Presidencia de la República y sin la vigilancia de la Contraloría General, por contar dicha dependencia con su propio presupuesto, se inician las primeras gestiones para adquirir los equipos requeridos para dar marcha al proyecto. El General Rojas Pinilla recibía de Arango, la certidumbre de celebrar su primer año de gobierno con la inauguración de tan codiciado y potente invento.

Fernando Gómez Agudelo tenía solo ocho meses para cumplir el deseo del presidente. Viaja entonces de inmediato a Boston donde su hermano Ricardo, doctor en física del Massachusetts Institute of Technology-MIT-, para contactar un grupo de expertos en radiodifusión que estudiarían cuál sería el mejor sistema de transporte de señal en un país como Colombia, con una topografía muy compleja.
Las conclusiones de ese rápido e improvisado estudio aconsejaban que la tecnología requerida no se encontraba en Estados Unidos sino en Alemania. Gómez Agudelo, entonces, viaja a Europa y adquiere los equipos. Quedaba aún por definir, dónde se pondrían los mismos para cubrir la señal que se transportaría desde Bogotá y Manizales, dónde se instalarían los estudios, qué se programaría, cómo se emitiría, es decir lo que se necesitaba para poner en funcionamiento una estación, un sistema de televisión, en un país que no tenía profesionales formados o conocedores de la televisión.

\footnotetext{
“...Completados los estudios y escogidos los sistemas, en diciembre de 1953 Gómez Agudelo fue encargado oficialmente por Arango de la puesta en marcha e instalación de la televisión, la cual debería inaugurarse exactamente el 13 de junio de 1954...”
}

El primero de mayo de 1954 se realizó la prueba de transmisión entre Bogotá y Manizales. Posteriormente, en el proceso de montaje, se continuó con la instalación de los primeros estudios de producción, simultáneamente avanzaba la preparación del primer grupo de profesionales colombianos, artistas empíricos, jóvenes universitarios, escritores y técnicos de la radio, que harían parte de aquella naciente televisión.

Aunque se estaba conformando un ejercito de realizadores, técnicos y productores de televisión, el problema de Gómez Agudelo era que necesitaba para salir al aire gente que tuviera experiencia, por eso viaja a Cuba en busca de productores, directores y camarógrafos. Gómez Agudelo necesitaba un grupo humano que le asegurara cumplir el propósito del presidente.

Dice el autor del artículo "Precursores y Pioneros" publicado en el libro "Historia de una travesía. Cuarenta años de la televisión en Colombia" que:

\begin{abstract}
“...por un olvido ocasionado por la precipitación de los acontecimientos, con la instalación de la televisión nacional nació simultáneamente el sistema de producción colombiano, que es único, quizás en el mundo de la televisión. Cuando Fernando Gómez Agudelo contrató el personal en Cuba, omitió un elemento vital en la producción de un programa de televisión: el director. Gaspar Arias era director de cámaras, pero no de producción, y cuando se fue a hacer la primera transmisión, Arias inquirió por el director y al no existir tal elemento en Colombia, hubo que optar por confiarle la dirección del programa al director de cámaras (switcherenginner), quedando establecida desde ese instante la presencia del director en la cabina de producción...”
\end{abstract}

Además de estar unida al poder, de ser oficial, de ser centralista, de definir en el papel que sus objetivos son la educación y la cultura, la historia nos muestra que el nacimiento de la televisión en Colombia fue improvisado. Ocho meses para 
su montaje, y 8 días para el estudio. Un viaje acelerado a otro país para la consecución del personal de producción y, la administración, producción, programación y montaje a cargo de una oficina del gobierno, son apenas el signo del "parto prematuro" de la televisión en Colombia. Sin embargo el 13 de junio de 1954, a las 9 de la noche, Rojas Pinilla inauguraba el servicio de televisión en el país.

Al día siguiente, el 14 de junio de 1954, el periodista Álvaro Monroy del periódico El Espectador cuenta en el desarrollo de la noticia:

"Con el primer programa de anoche se ha despertado fiebre de televisión”, y agrega:

\begin{abstract}
“...Una de las personas más satisfechas con la inauguración de los canales 8 y 10 de la televisión oficial, fue el señor presidente de la República, quien inmediatamente terminó su discurso se trasladó a sus habitaciones particulares, a ver el desarrollo del programa...
\end{abstract}

Se entrevistó al productor cubano Gaspar Arias y esto contestó.

-¿Qué impresión tiene del primer programa televisado?

-¡Oye chico!, yo estoy muy satisfecho de esto. Claro que hay muchas cosas que se pueden corregir, pero la cosa fue que salimos el 13 de junio. Creo que había cierta indiferencia del público, porque nadie realmente creía en la televisión, pero vas a ver que se va a despertar 'fiebre de televisión'. ¡Palabra! Lo mismo que en todas las ciudades.

$-¡ Y$ el personal?

-¡Hombre! Estoy seguro de que el personal colombiano en poco tiempo estará haciendo cosas magníficas..."

Escribe más adelante el periodista:

“...Al señor presidente -dijo Arias- lo televisamos desde todos los ángulos y salió muy bien...”

Avanza la noticia:

“...El Ministro de Relaciones Exteriores, Evaristo Sourdís, declaró, luego del discurso del presidente, que la televisión debía ser estatal y por ende cultural en un ciento por ciento. Sin embargo, se ha sabido que es posible que el gobierno venda algunos programas comerciales..."

Para expresar más aún el carácter oficial del medio y el acento propagandístico del General Rojas Pinilla en la pantalla, durante varios meses la presentación de planta mostraba en un plano la siguiente composición: con un telón de fondo, a la izquierda sobre una cámara el perfil del General, y al lado derecho, en un nivel más bajo que él, un cuadro del libertador de Colombia Simón Bolívar.

Para enero de 1955, el decreto 0101, crea la Televisión Nacional, buscando organizar o darle cuerpo a ese principio de oficina de televisión adscrita a la Radiodifusora Nacional, que salió a las carreras y con improvisación. Después de dos años de fundada, la Televisora Nacional extendía sus redes, ya llegaba con su señal a Medellín y a las ciudades capitales del Eje Cafetero y al Valle del Cauca. El 10 de mayo de 1957, el presidente Gustavo Rojas Pinilla es depuesto, y una Junta Militar asume el control del Gobierno. La Televisora Nacional sigue funcionando con el mismo esquema de programación y contratación.

Un hecho insólito ocurre el 19 de diciembre de 1957, el director de la Televisora Nacional anuncia que entre el 1 y el 11 de enero de 1958 se apagará la red para someter a todos sus equipos a reparación. La circular del Director, entre otros anuncio dice:

\begin{abstract}
“...para dar cumplimiento a los programas comerciales, cuyos contratos están vigentes a la fecha, la Televisora Nacional descontará de la cuenta del mes de enero el valor de los espacios que deberían realizarse durante los días antes mencionados...”
\end{abstract}

\section{Modelo mixto de televisión}

A mediados de agosto de 1955, ocurre un hecho que marcaría en lo sucesivo la televisión colombiana, se daría paso al modelo de televisión mixto, único en América. El Gobierno Nacional decide en agosto de 1955, al año y dos meses de su nacimiento, delegar a la empresa Televisión Comercial- TVC-, una sociedad de las empresas radiales Caracol y Radio Cadena Nacional, RCN, la explotación comercial de algunos espacios en la programación del canal nacional.

En noviembre de 1955 en el boletín de programas de la Radiodifusora Nacional, en el artículo "La TV como vehículo cultural", se justifica la apertura de la televisión estatal y el arriendo de los espacios a particulares, así:

“...Con miras a este mejoramiento de nuestros servicios, el Gobierno Nacional estimó conveniente el ingreso de algunos elementos de competencia en el campo de la TV y, en consecuencia le abrió las puertas a la TV comercial.

Con respecto al funcionamiento de la TV comercial conviene aclarar que el Estado no renuncia al control y supervigilancia de cuantos programas se transmitan a través de la Televisora Nacional.

De aquí que en esta se admitan anunciadores y no patrocinadores, toda vez que el significado y sentido de este vocablo implica el ejercicio de funciones de las cuales no ha renunciado ni podría renunciar el Estado, sin perjudicar los intereses de la comunidad que se beneficia con la TV. El anunciador alquila el tiempo y suministra los programas, pero el Estado se reserva el derecho de administrarlos o rechazarlos, según que favorezcan o contraríen los intereses de la audiencia colombiana. 
Hay una evidente diferencia entre aceptar anuncios y aceptar patrocinadores. La prensa, por ejemplo, acepta anuncios, pero no renuncia a la posibilidad de los juicios $\mathrm{u}$ opiniones emitidas en sus informaciones, noticias, comentarios y columnas editoriales. Las empresas de cines anuncian al comienzo y en los intermedios de sus exhibiciones de películas, pero sus programas no son patrocinados por los anunciadores.

Se ha sugerido que mientras a los anunciadores no se les permita patrocinar, esto es, controlar los programas de TV que salen al aire con sus avisos, la televisión será poco halagadora para ellos como eficaz vehículo de propaganda. Las directivas de la Televisora Nacional han consultado este punto con sus distintas casas y agencias de publicidad, llegando a la conclusión de que esa relativa independencia entre el anunciador y su programa, no perjudica en nada el éxito financiero del nuevo servicio de la TV Nacional..."

Como quien dice, el problema principal de la naciente televisión colombiana era la financiación. El Estado arrienda algunos espacios, pero como lo dice su boletín oficial, estaba claro que con esta práctica no renunciaba al control delos contenidos. Se extendió el boletín en explicaciones. El Ministro de Relaciones Exteriores, de la época, Evaristo Sourdís, al respecto, el 14 de junio de 1953, un día después de realizada la primera emisión, señaló que en el diseño de dicha televisión no se había contemplado como responsabilidad del Estado el costo de operación del sistema. O mejor, se contempló que lo haría la empresa privada.

Quienes conocen de televisión, afirman que el montaje técnico de un canal es lo de menos, los equipos de transmisión y producción se consiguen en el mercado de acuerdo con un presupuesto determinado, la factibilidad técnica de una estación de televisión con el soporte de un buen estudio, debe dar buenos resultados. Pero, qué decir de su programación, cuál es su orientación, cuánto cuesta alimentarla y producirla, esto fue lo que poco pensaron quienes dieron marcha a la televisión en Colombia.

A cuatro meses de la apertura comercial, el 4 de diciembre de 1955, en entrevista concedida al periódico El Espectador, el Gerente Encargado de TVC, Fernando Londoño Henao, directivo de Caracol, declaraba que la televisión comercial había sido un éxito desde el punto de vista de la programación:

“...como pueden apreciarlo, se ofrecen magníficos programas y sólo faltan dos o tres espacios para copar las dos horas cedidas por el Gobierno Nacional...”

Dicho modelo de Tv era un buen negocio para los particulares, no se podía entender de otra manera que las dos más grandes cadenas radiales, dieran un buen parte. Así, con la creación de TVC, desde sus inicios la oferta de programación de la televisora colombiana mezclaba en su parrilla unos programas producidos por la oficina de la Televisión Nacional, con la oferta que ya era creada y explotada comercialmente por particulares. La experiencia de TVC se prolongó hasta finales de $1956 \mathrm{y}$ :

“...sirvió de base para que se iniciara otra modalidad de comercialización de la televisión colombiana, que se extendió durante aproximadamente diez años: el arriendo directo de espacios (obviamente los de mayor sintonía) a la Televisora Nacional por parte de agencias publicitarias para producir programas, muchas veces exclusivos para sus clientes... Junto a ese grupo de agencias, nació más adelante otro más pequeño, constituido por empresarios que le arrendaban a la Televisora Nacional determinados espacios, producían sus programas y luego los financiaban o trataban de financiarlos con anuncios publicitarios de la industria y el comercio..." [1]

En ese principio de oferta mixta, la programación a cargo de los particulares se destacaba claramente, qué programa no era de la Televisora Nacional. Veámoslo en los siguientes anuncios de la programación de la época:

\section{Cuadro No.1}

Anuncio de la programación de la época

\begin{tabular}{|c|c|c|c|}
\hline Horario & Martes: & Miércoles: & Jueves \\
\hline $6: 45 \mathrm{pm}$ & $\begin{array}{l}\text { Dulces } \\
\text { Navidades }\end{array}$ & & \\
\hline 7:00 pm & $\begin{array}{l}\text { Los postres } \\
\text { Royal } \\
\text { Preguntan }\end{array}$ & $\begin{array}{l}\text { La barra } \\
\text { infantil }\end{array}$ & $\begin{array}{l}\text { El Club del } \\
\text { Tío Alejandro }\end{array}$ \\
\hline $7: 30 \mathrm{pm}$ & $\begin{array}{l}\text { Guillermo el } \\
\text { incomprendido }\end{array}$ & $\begin{array}{l}\text { Telepáticos, } \\
\text { chiclets } \\
\text { Adams }\end{array}$ & $\begin{array}{l}\text { Los alegres } \\
\text { cocineros }\end{array}$ \\
\hline $7: 45 \mathrm{pm}$ & $\begin{array}{l}\text { Aguinaldo } \\
\text { Sendas }\end{array}$ & & \\
\hline $8: 00 \mathrm{pm}$ & $\begin{array}{l}\text { Revista } \\
\text { Musical } \\
\text { Sedeco }\end{array}$ & $\begin{array}{l}\text { Programa } \\
\text { Pielroja }\end{array}$ & $\begin{array}{l}\text { Programa } \\
\text { de la Esso } \\
\text { Colombiana }\end{array}$ \\
\hline $8: 30 \mathrm{pm}$ & $\begin{array}{l}\text { El minuto de } \\
\text { Dios }\end{array}$ & $\begin{array}{l}\text { Contesta y } \\
\text { Dana paga }\end{array}$ & $\begin{array}{l}\text { Descubra el } \\
\text { Secreto }\end{array}$ \\
\hline
\end{tabular}

Cuando el gobierno de Rojas Pinilla autoriza el funcionamiento dela Televisión Comercial-TVCse da inicio a un sistema que hasta 1998 cuando aparece la televisión privada, se caracterizó por la escasa y esporádica financiación de la televisión pública por parte del Estado.

Modelo mixto que funcionó, de un lado, con unos particulares quienes con el beneplácito del Estado, dueño de costosas redes de transmisión, explotaron comercialmente el medio en los horarios de mayor sintonía desde su nacimiento, incluso definiendo con el correr de los años, qué se quería ver. Y del otro lado, un Estado que abandona de su responsabilidad educadora y de 
su promoción cultural, en una actitud sistemática desde su génesis. Posteriormente a esos diez años de sucesivo arriendo de espacios a agencias y empresas privadas, el modelo se perfeccionó. Se estableció que mediante la figura de la licitación pública, la Televisora Nacional adjudicaría a los licitantes, espacios de televisión por un término que podría renovarse según se definiera en el pliego.

En este desarrollo y perfeccionamiento del modelo, las empresas, ya productoras de televisión, se inscribían en la Televisora Nacional y con ello adquirían el derecho a participar en las sucesivas licitaciones. Mientras el Estado crecía la red de transmisión con sus respectivos costos, los particulares crecían sus ganancias. Y la programación, la continuaban conformando la oferta comercial de los particulares en los horarios de mayor sintonía, mientras la oferta educativa, la realizaba la Televisora Nacional en los restantes horarios. Era evidente la protección del Gobierno a los particulares.

En su momento este hecho, generó la versión sobre la venta o alquiler de dicho ente:

\begin{abstract}
“...En cuanto a las versiones que han circulado últimamente sobre venta o alquiler de la Televisora Nacional a particulares, nada nuevo ha habido sobre el particular, con excepción de una presunta propuesta al Gobierno Nacional por parte de la cadena radial Caracol y de Radio Cadena Nacional, entidades cuyos dirigentes están estudiando varias fórmulas para presentárselas al Gobierno...”[2]
\end{abstract}

Este hecho en apariencia insignificante, muestra el inicio de una pugna sucesiva por el control del medio. Un Estado dueño de la frecuencia y de los canales, que alquila a particulares. Particulares, "inquilinos" de unos espacios, que aspiran a su vez, a controlarlos en su totalidad. Un Estado que no quiere soltar la operación y unos particulares que quieren dominar el negocio, sin hacer la inversión en el montaje y mantenimiento de la red. Hecho sucesivo hasta 1998, cuando inician transmisión las únicas dos cadenas privadas de televisión actualmente existentes en Colombia, que "paradójicamente" en la actualidad son de propiedad de los grupos de telecomunicaciones Caracol y Radio Cadena Nacional, cuyos canales se llaman Caracol Televisión y RCN Televisión.

Mientras tanto la red crecía. Para 1961, se tendría cubierto las ciudades capitales y algunas poblaciones de los Santanderes, Cauca, Tolima, Huila, Boyacá, Chocó, Nariño y la Costa Atlántica. Prácticamente se llegaba con la señal a las zonas Pacífica, Atlántica y Andina. Y con la extensión de la red, crecían las ganancias de los particulares, mientras tanto la Televisora Nacional continuaba recibiendo dinero por el alquiler y un mínimo dinero del presupuesto nacional para su financiamiento. Y sobre el cumplimiento de su objetivo como medio para la educación y la cultura, con lo que le generaban estos ingresos, se permitió el funcionamiento del Departamento de Televisión Educativa, creado en junio de 1960.

\section{Televisión educativa}

Decía el proyecto que lanzaba el Departamento de Televisión Educativa en 1960, que en el caso particular de Colombia, éste serviría para perfeccionar los métodos educativos que se emplean en muchas escuelas. Después de varios meses de preparación, en abril de 1961 se emitió la programación educativa, se dictaron en los horarios de la tarde, teleclases de matemática, biología, música y geografía para niños de educación básica. Un hecho que una vez más da cuenta de la improvisación y falta de una política estatal del medio, lo hayamos en el texto "Historia de una Travesía. Cuarenta años de la Televisión en Colombia" que informa, después de la sonada inauguración de la televisión educativa en abril de 1961, que:

\begin{abstract}
“...Con el objeto de consolidar más el proyecto de televisión educativa y de llevarlo a una escala nacional, en el más breve tiempo posible, se elaboró un decreto que entrará a regir próximamente, por medio del cual se crea una Comisión Nacional de Televisión Educativa, integrada por el señor Ministro de Educación o su representante; el director técnico del Ministerio de Educación; el rector de la Universidad Nacional o su representante, y el director de la Radiotelevisora Nacional. El decreto vincula en forma definitiva al Ministerio de Educación a este proyecto y lo autoriza para nombrar el personal correspondiente..."
\end{abstract}

La Televisora Nacional creaba una programación educativa formal, un microsistema de tele-escuela, pues solo cubría ciertos grados y materias de la educación básica primaria, sin la presencia del Ministerio de Educación en su fase de planeación.

El Ministerio de Educación lo vinculan para la ejecución. Según se lee en texto citado, para consolidar el proyecto de televisión educativa se crea con posterioridad una comisión de la cual haría parte el Ministerio de Educación. Este hecho, una vez más muestra la improvisación, y otra faceta, la falta de una política de televisión educativa en Colombia.

Aunque pudiera entenderse y hasta justificarse esta situación ocurrida en 1961, en donde podría argumentarse la falta de experiencia en asuntos de televisión, para confirmar la tesis de que en Colombia no existe una política en materia de televisión y menos aún de televisión educativa, solo se han hecho intentos por formularla, en 1997, muchos años después de la extinción de la propuesta de tele-escuela, se habla de crear un canal educativo en el cual se repite la historia: nuevamente no participa el Ministerio de Educación. 
En 1962 el gobierno de entonces, ante la potencialidad ofrecida por el medio, y ante el éxito en el cubrimiento de la propuesta educativa, solicita al gobierno de Estados Unidos financiar la realización de un plan piloto. Esta ayuda se esperaba recibir del programa de gobierno norteamericano La Alianza para el Progreso. Dicha propuesta se planteaba para lograr un cubrimiento nacional, diseñaba el desarrollo de dos ciclos, el primero, con una duración de 18 meses, en cuyo periodo se prepararía al personal que ejecutaría la enseñanza por televisión. El segundo, proponía extender la señal a las escuelas.

Cuando en los primeros años se hablaba de televisión educativa, pese a la inconsistencia de excluir en la fase de planeación al Ministerio de Educación, con la inyección de capital extranjero, el proyecto de tele-escuela creció y se fortaleció, $y$ fue reconocido posteriormente en América Latina como experiencia piloto.

Las franjas de televisión educativa formal se estructuraron en tres secciones, una, escolar, otra, para adultos y una más, de extensión cultural. La franja escolar presentaba cursos para niños de primero, tercero y quinto de primaria, en las materias de matemáticas, sociales, ciencias naturales, música, dibujo y deportes. La programación educativa para adultos se propuso capacitar a los maestros. La franja de extensión cultural, que en algunos días ocupaba las horas comerciales, tenía como finalidad la enseñanza de las bellas artes, con la presentación de programas musicales, de teatro y pintura.

\subsection{El teleteatro}

En ese modelo híbrido de contratación y programación por el que optó la televisión en Colombia al entregarle sus espacios estelares a las propuestas comerciales, y la de emitir programación educativa escolar en las franjas de la mañana y la tarde, se suma el desarrollo de una propuesta de programación cultural, entre ellas la de mayor inversión para el Estado: el teleteatro.

Desde 1955 el espacio cultural dedicado al teatro por la Televisora Nacional, fue al decir de muchos "la niña mimada". El presupuesto conseguido con el arriendo de los espacios y con el asignado por el Estado, permitió financiar dicha propuesta. Del pago de la nómina dedicada a los artistas y talentos, el 70 \% la absorbía el teleteatro. Con el teleteatro, se formaron libretistas, directores y artista que hicieron escuela y en años posteriores, dieron origen a proyectos y producciones dramatizadas que les han valido a las realizaciones colombianas el reconocimiento en el ámbito latinoamericano.

\section{Primeros años de la televisión regional}

Como una necesidad de expresión cultural de las regiones, en un país que por 30 años vio una televisión centralista en su producción y contenidos, nace el primer canal de televisión regional en Colombia, Teleantioquia. Excepto los bogotanos, los televidentes del país no veían reflejado en la televisión que recibían una realidad diferente a la de la ciudad capital de Colombia, o una interpretación diferente del país que la que se hacía desde allí. Las regiones eran motivo de aparición en los dos canales de cubrimiento nacional, sólo cuando sucedían hechos noticiosos que lamentar, o cuando éstos se vinculaban con la vida política de dirigentes regionales de trayectoria nacional.

Por eso desde los años 70, crecía el número de voces que reclamaban una programación que reflejara el sentir y las diferentes manifestaciones culturales de las regiones. En esta década, en un contexto político nuevo para Colombia, crecían también las voces que clamaban un país menos centralista.

En 1978, en medio de un nuevo orden político y social del país, se presenta para aprobación del Congreso de la República, el primer proyecto de ley de televisión en Colombia, y en este se proponía autorizar la creación de los canales regionales de televisión. Desde el gobierno liberal en el poder, se argumentaba a favor del proyecto. Se decía que:

\begin{abstract}
“...la televisión regional no supondría competencia entre las programadoras que trabajaban con los canales nacionales -dado el centralismo de éstos- $y$ que la descentralización evitaría el control por parte del ejecutivo..."
\end{abstract}

Agregaba el Gobierno Nacional que con la aparición de la televisión regional la programación de los canales mejoraría, se generarían nuevos puestos de trabajo y se contribuiría con el desarrollo regional. Debían, los ponentes del proyecto, romper la resistencia de las programadoras bogotanas, quienes veían en los canales regionales una competencia que les robaría parte de su tajada publicitaria. Para esa época se suponía, que los canales regionales replicarían el modelo de la televisión nacional. Vale la pena recordar que las programadoras de los dos canales nacionales para la época, eran empresas productoras de televisión que tenían con INRAVISION contratos de concesión de programas en los mismos, ejem: Caracol, RCN, Punch, RTI, JES, entre otras.

Igualmente justificaba el gobierno, ante la oposición en Bogotá, que dichos canales por no estar centralizados evitarían el control por parte del ejecutivo. Implícitamente se reconocía la 
intromisión del gobierno de turno en la televisión, y a la par se desconocía públicamente la necesidad de descentralizar en sus contenidos dicha televisión. El proyecto de ley no fue aprobado en el Congreso, entre otras razones, por la presión de los programadores para que no se diera vía libre a la televisión regional. Con la caída del proyecto de ley, también se perdía la posibilidad de que la operación de la televisión tuviera un marco legal. Lo cual convenía de alguna manera a ciertos contratistas que querían seguir sacando dividendos económicos del caos como se producía y emitía televisión en Colombia.

Para 1979, en medio de la derrota, las presiones $\mathrm{y}$ voces de las regiones que clamaban por verse en la televisión no se habían extinguido. El 5 de mayo de 1979 según reseña la prensa, en un aparente sorpresivo viraje del Gobierno Nacional, esos reclamos recibieron del Ministro de Comunicaciones José Manuel Arias Carrizosa la siguiente respuesta a sus expectativas:

\footnotetext{
“...En cuanto a la participación de las regiones en la programación y en el orden a ése propósito, hemos proyectado y estamos diseñando un programa que se llamará Colombia y estará hecho básicamente con la presencia de cada una de las regiones del país. Se tomarán y mostrarán todos los aspectos del orden departamental, sin ningún tipo de limitaciones, pero desde luego, haciendo televisión y buscando que Colombia se integre un poco más dentro de un propósito que yo diría es obsesivo del Gobierno Nacional como es el de crear factores de integración del país...”
}

Así respondía el Gobierno Nacional a la necesidad cultural de las regiones. No parecía "muy obsesivo" en comprender la argumentación desde las mismas. Con un programa de televisión, creía, resolvía de un plumazo el problema de la desaparición en televisión, de toda la otra Colombia que no era Bogotá.

El español Miguel Ángel Gea Rifà en su libro “Televisión regional y desarrollo, aproximación a la experiencia colombiana" argumenta, que en el rechazo del Ministro subyace la concepción centralista del Estado Colombiano plasmada en la Constitución de 1886, la que rigió en Colombia hasta 1991. Agrega el español, que con el rechazo del gobierno en cabeza del Ministro Arias Carrizosa, a la creación de canales regionales

“...se percibía desde el Gobierno como un elemento disgregador y no como un instrumento capaz de favorecer la identidad mediante el reconocimiento y la potenciación de la diversidad...”

Conel rechazodelCongresoala propuestaLeyycon la posición del Gobierno Nacional, continuarían pues las regiones sin un referente visual que les permitiera identificarse con su entorno inmediato y sin la posibilidad de expresarse. Tendrían que continuar viéndose esporádicamente, sólo en hechos de orden público. Dice Fernando Calero Aparicio, exdirector de INRAVISIÓN y exgerente de Telepacífico, en su crónica

"Los Canales Regionales: trayectoria y perspectivas", que INRAVISIÓN como institución de un estado centralista, no entendió a su debido tiempo “...que el desarrollo de la televisión tenía precisamente una necesidad de arraigamiento también regional y local..."

\subsection{Los 80 y 90, explosión de canales regionales}

Al iniciar la década de los 80, las demandas para que se crearan canales regionales crecieron, y dice en la crónica citada el exgerente de Telepacífico, que fueron las de los antioqueños, costeños y vallecaucanos, las que más se oían clamar por la creación de canales de televisión que estuvieran cerca del sentir regional. En el Departamento de Antioquia, a pesar de la negativa del Gobierno Nacional, las universidades, y 2 entidades descentralizadas del orden departamental, el Instituto para el Desarrollo de Antioquia IDEA- y las Empresas Departamentales de Antioquia -EDA- hoy EDATEL, actuales socios de Teleantioquia, seguían promoviendo en la región la necesidad de fundar un canal regional de televisión.

Para el país, el nacimiento de la televisión regional era un hecho trascendental, y respecto a la significación cultural y política, y a su deber ser, dice al abogado Jaime Abello Banfi, exgerente de Telecaribe, en su crónica La Televisión Regional en Colombia que:

\footnotetext{
“...los canales regionales de televisión tienen, por lo menos, cuatro misiones en relación con el hecho sociopolítico de las regiones: buscar la integración del territorio de una red televisiva con cobertura total sobre la región servida; dar prelación a la producción televisiva de origen regional, a la reafirmación de la identidad cultural y al tratamiento de los temas y de la información regionales dentro de la programación habitual; consolidar una industria televisiva y un mercado publicitario de alcance regional y, finalmente, comprometerse con la suerte de las comunidades territoriales respectivas y con el proyecto político de autonomía territorial y de regionalización...”
}

Para los años 80 era un hecho que en Colombia, un país de regiones, el nacimiento de la televisión regional no podía pararse pese al deseo de unos pocos que veían en el medio, sólo un negocio. 


\subsubsection{Teleantioquia}

\section{Teleantioquia En 1984, durante la presidencia}

del antioqueño, Belisario Betancur Cuartas, se vivía en el país un ambiente político diferente, se tomaban una serie de medias descentralistas, entre otras decisiones importantes para la vida política de los colombianos, se aprobaba la elección popular de alcaldes. No podía ser en otro contexto que el descentralista, cuando finalizando 1984, el presidente Betancur, expide dos decretos relacionados con la televisión: el Decreto Nacional 3100 que autoriza la creación de los canales o cadenas regionales de televisión, y otro, el 3101, en que se autoriza a INRAVISIÓN a firmar un contrato de sociedad con las Empresas Departamentales de Antioquia - EDA- para establecer en Antioquia un canal regional.

Registraba la prensa, que Antioquia sería el primer Departamento del país en contar con un canal regional, informaba, que en seis meses los antioqueños podrían ver su propia televisión. Con la firma de los decretos, se cumplía así el sueño de las regiones, verse reflejadas en la televisión.

Ver a las regiones en este medio de una manera diferente, con una mirada que no fuera centralista o simplemente la de los titulares amarillistas. El Decreto Nacional 3101, constituía la primera sociedad de televisión, Teleantioquia, cuya estructura contaba con un Consejo Directivo Regional de Televisión, quien hacía las veces de Junta Directiva.

Dicho Consejo estaba conformado por el Ministro de Comunicaciones, quien lo presidía, el Gobernador de Antioquia, el Gerente de las Empresas Departamentales de Antioquia, EDA, y el Rector de la Universidad de Antioquia, quienes nombrarían al Gerente, que sería un funcionario de libre nombramiento y remoción, a quien correspondía dirigir la operación del canal. Con la creación de Teleantioquia el 11 de agosto de 1985, ocho meses después de firmados los Decretos Nacionales 3100 y 3101, renace pues la esperanza en el Valle del Cauca y en la Costa, regiones que venían discutiendo la necesidad de formar sus propios canales.

Como pionero de la televisión regional en Colombia, a Teleantioquia le tocó desde un principio afrontar las limitaciones impuestas a una estación, que se autorizó funcionar como un canal educativo y cultural, sin grandes capitales para la operación y sin autorización para la comercialización.

\subsubsection{Telecaribe}

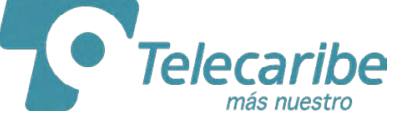

En abril de 1984, más nuestro

cuando aún no se había firmado el decreto que dio origen a televisión regional, el Centro de Estudios Periodísticos de la Costa, organizó en Barranquilla un foro al que fueron convocados los diferentes sectores que discutían desde años atrás, la idea de crear para la región un canal de televisión. En dicho foro, se concluyó que para la zona era necesario rescatar la identidad cultural a través de este medio.

Allí también se planteó como necesidad, que en el canal de la región estuvieran asociados todos los Departamentos, así como también que dada la condición de canal público, su programación debería ser cultural y educativa. En el foro se constituye entonces, el "Proyecto de Creación del Canal regional de la Costa Atlántica”, cuyo objetivo era definir las condiciones financieras, técnicas y de programación del futuro medio.

Con este precedente, a menos de un año de fundada Teleantioquia, exactamente a dos años de realizado el foro, nace en abril de 1986, Telecaribe, el canal que reúne los 6 departamentos de la región de su mismo nombre, son ellos: Guajira, Cesar, Sucre, Magdalena, Atlántico, Bolívar y Córdoba.

Telecaribe se crea como una sociedad conformada por INRAVISIÓN, el Departamento de Atlántico, la Universidad de Cartagena, el Instituto de Cultura y Turismo del Cesar, el Instituto Financiero para el Desarrollo de Córdoba, INFICOR, la Corporación Departamental de Turismo de Guajira, el Instituto de Cultura de Magdalena y el Instituto para el Desarrollo de Sucre. El aporte de cada uno de los socios fue de $\$ 100.000$.

Para los "costeños", como se les dice a los pobladores de esta región, Telecaribe nace como un canal que incluye en su programación producciones que reflejan manifestaciones culturales y deportivas, no muy comunes en el resto del país. El día de su inauguración, el 28 de abril de 1986, los habitantes de la Costa Atlántica, disfrutarían una programación especial originada desde Valledupar. Quedaba atrás el transistor, muchos costeños verían por primera vez el festival de música vallenata.

La sede donde inició sus emisiones Telecaribe fue Valledupar, capital de Cesar, pues el canal local Televallenato, que funcionaba desde 1984 sin la debida licencia, le dio la base tecnológica para operar. A los pocos años, ésta se traslada a Barranquilla, la ciudad con mayor desarrollo de la región. Este canal, empieza a emitir su señal para toda la región, tanto en banda UHF como 
en VHF, dependiendo de la ubicación de las estaciones repetidoras.

Telecaribe, desde sus inicios encara dos (2) retos tecnológicos nunca vistos en el país, uno, extender su red en dos (2) bandas y dos, montar un sistema que permitiera las emisiones descentralizadas. Por ser el caribe colombiano una región extensa que reúne pobladores de diferentes idiosincrasias, le correspondió ser el pionero en Colombia de las emisiones descentralizadas. En su programación contempló desde sus inicios, realizar una propuesta con emisiones para los noticieros, originadas desde las principales ciudades capitales de la zona: Cartagena, Santa Marta, Montería, Sincelejo y Valledupar.

Con la condición tecnológica de emitir en dos bandas, Telecaribe se vio obligado a realizar un esfuerzo mayor para conseguir su audiencia, ya que hasta la fecha de su inauguración toda la televisión del país se veía en banda VHF.

\subsubsection{Telepacífico}

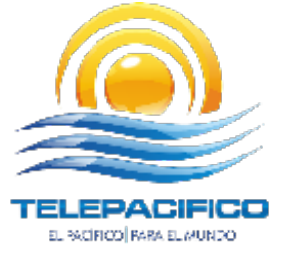

Con la experiencia acumulada por Teleantioquia y Telecaribe, el 8 julio de 1988 nace Telepacífico. Canal regional de la zona pacífica colombiana. Esta región agrupa los Departamentos de Chocó, Valle del Cauca, Cauca y Nariño. Telepacífico estaba autorizado por la ley para cubrir los cuatro departamentos, y al momento de su constitución, 1986, las empresas socias eran INRAVISIÓN, el Instituto financiero para el desarrollo del Valle del Cauca -Infivalle- y el Departamento del Valle, lo cual en la práctica demostraba que el Canal era sólo del y para el Valle del Cauca.

Cuando el 25 de julio de 1986 se asocian estas entidades se crea la sociedad de televisión, Televalle, que posteriormente sería Telepacífico. Si bien Teleantioquia inicia en 1985 su programación con la condición particular de asumir el total de la oferta, Telepacífico, analizando la experiencia del canal de Antioquia, en cuanto a las dificultades financieras presentadas en su primer año de funcionamiento, $\mathrm{y}$ acogiendo el decreto 2683 de 1986, inicia su operación con 8 empresas productoras.

Para el Gobierno Nacional y en particular para el Ministerio de Comunicaciones regente de la política de televisión en Colombia en 1986, era claro que ni Teleantioquia, ni ningún canal regional naciente, era capaz de sostenerse. Por eso mediante el decreto 2683 autoriza la comercialización de los canales regionales y la aparición de contratistas privados. A un año de fundado el primer canal regional se repetía la historia de la televisión nacional. Se instaura un modelo mixto para la televisión regional, se copiaba el modelo nacional.

En 1985 la televisión regional nacía como un medio cultural al que no le autorizaban ningún tipo de comercialización, y Telepacífico nacía con el camino despejado para hacerlo. Según Fernando Calero Aparicio, exgerente del canal, Telepacífico se preocupó por fomentar una política informativa independiente, pluralista, responsable y descentralizada a través de toda su propuesta de programación generalista.

Para 1988, más que ninguno de los dos canales regionales existentes, Telepacífico entendió como elemento clave para conquistar su audiencia en un medio naciente, que no contaba con la credibilidad de sus nuevos televidentes, que la presentación de producciones regionales en directo, sería su ventaja competitiva. Así, durante los primeros años de creación del Canal, se vieron los festivales musicales que congregaban a la región, las festividades populares de los municipios del Departamento, la programación cultural de la región, en fin, los personajes y las manifestaciones culturales que hacían notar a los vallecaucanos, la diferencia frente a una televisora nacional que no las cubría.

En 1992, la señal dejaba de ser exclusiva para Cali, ciudad capital del Valle del Cauca, y municipios vecinos. Telepacífico extiende su señal a los municipios del norte del Valle, a Popayán, capital de Departamento del Cauca y a algunos municipios de dicho territorio. Por fin Telepacífico dejaba de ser sólo para el Valle del Cauca. Para 1993, los Departamentos del Cauca, Nariño y Chocó, reclamaban que la señal de Telepacífico, como canal de la región, llegara a sus localidades.

Sólo en 1999 después de realizar grandes inversiones, y con el apoyo del sistema satelital, ejecuta su proyecto de expansión de la señal. Para este año, Telepacífico cubría el territorio del Departamento del Valle del Cauca en un 99\%, el del Departamento de Cauca en un $80 \%$, el de Nariño en un $80 \%$ y el de Chocó en un $70 \%$.

Durante sus años de operación han cubierto en directo eventos culturales característicos de la región, tales como: Festival musical del Mono Nuñez, Festival de Bandas de San Pedro, Festival Petronio Álvarez, Fiestas de la Caña, Festival de Blancos y Negros de Pasto, en fin, una serie de fiestas populares que no aparecerían en pantalla, si no fuera a través de la televisión regional del Pacífico colombiano. 


\subsubsection{Telecafé}

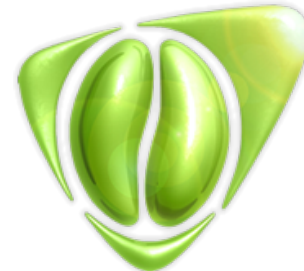

Después de ocho años de espera en la región cafetera de Colombia, el 17 de octubre de 1992, nace Telecafé. La Sociedad Canal de Televisión Telecafé, se formaliza en 1990 y tiene como socios a INRAVISIÓN y los departamentos de Caldas, Quindío y Risaralda. Más tarde se incorporan a la sociedad, las Empresas Públicas del Manizales y la Universidad de Quindío. La zona de influencia del Canal son los tres departamentos asociados, los cuales forman el eje cafetero de Colombia, y hacen parte del grupo sociocultural paisa. En esta región conformada por Caldas, Quindío y Risaralda, históricamente, se han presentado disputas por el reconocimiento de la hegemonía cultural y política de sus ciudades capitales. Manizales, Armenia y Pereira, respectivamente.

Estos tres departamentos que hasta 1966, hacían parte de Antioquia, cuando decidieron independizarse, no lograron llegar a un acuerdo para nombrar cual de las tres ciudades sería el centro administrativo y político de lo que se suponía, sería una nueva y única unidad administrativa regional. Y ese mismo conflicto histórico se trasladó a su canal regional. En 1984, inician los primeros acercamientos para formular el proyecto; en 1990, acuerdan constituir la sociedad; y sólo dos años después, luego de unas negociaciones entre sus socios, pueden salir al aire.

Esta vez en la región, la disputa se dio por dos razones, primero, porque en esencia muchos pobladores no eran partidarios de crear un canal que uniera a los tres departamentos, y segundo, porque no lograban ponerse de acuerdo cual sería la sede del Canal y cómo administrarían la estación. Para poder dar vía libre al proyecto y zanjar de la manera más salomónica la disputa, acordaron que la sede se instalaría en Manizales, el Gerente sería de Pereira y el Subgerente de Armenia.

En sus ocho años previos a la primera transmisión, la cronología de su historia nos dice que, en 1984 y 1985 formulan el proyecto, en 1986 y 1987 realizan las primeras pruebas técnicas, en 1988 se firma el acta de intención de constitución del canal, en 1989 se nombra la primera gerente y, a comienzos del año 92, se realizan las primeras señales de prueba. Desde 1992 hasta 1994, Telecafé exploró una manera diferente de funcionamiento. A diferencia de Teleantioquia, Telecaribe y Telepacífico, no contaría con contratistas, este canal asumía la responsabilidad absoluta de la programación.
Decidió operar como muchos de los canales del mundo, programaría y comercializaría el 100\% de su oferta, la cual produciría o compraría a productores de la zona. No contó con éxito, la empresa comercializadora, Gente y Medios, le incumplió con los pagos, llevando al fracaso su propuesta y poniendo obviamente al Canal en un grave problema financiero. Después de este incidente, el Canal tuvo que recurrir al sistema mixto propio de la televisión colombiana, desarrollado ya para ese momento, con mayor fuerza en los tres canales regionales que le antecedieron.

\subsubsection{Canal Capital}

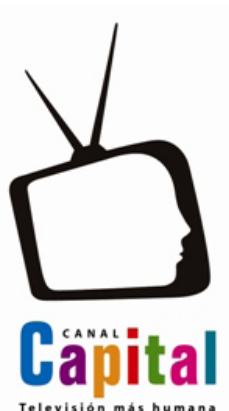

Este canal que cubre sólo el distrito capital, o sea la ciudad de Bogotá, nace por un Acuerdo del Consejo de Bogotá. El Canal, se crea como una sociedad constituida por el Distrito Capital, la Empresa de Telefonía de Bogotá, ETB, y el Instituto Distrital de Cultura y Turismo mediante escritura pública en 1995. La composición accionaria de Canal Capital se modificaría para enero de 2000, cuando la ETB vende su paquete accionario al Distrito Capital. Su programación empezó a emitirse en noviembre de 1998, bajo un lineamiento claro de ofrecer una programación mayoritariamente urbana, cuyo propósito era proyectar la imagen de la ciudad.

Cuando este Canal se planteó su filosofía de programación, concibió que su propuesta fuera no violenta y buscaría construir ciudad, para de esta manera contribuir con fortalecer la pertenencia e identidad en los habitantes de la ciudad capital, una de las de mayor desarraigo del país, pues la habitan gentes de toda Colombia. Paradójicamente, en un país acostumbrado a ver una televisión nacional capitalina, los habitantes de Bogotá no tenían hasta la fundación de Canal Capital, un medio de expresión cercano, por esto desarrolla desde sus inicios programación participativa.

\subsubsection{TV Andina - Canal 13 en la actualidad}

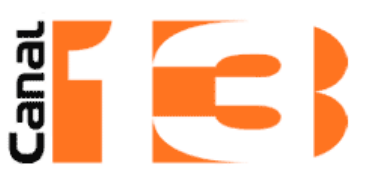

Constituida por escritura pública en 1995. Tv Andina, se erige como una sociedad conformada por

Telecom, los departamentos de Cundinamarca, Boyacá, Tolima, Huila, Meta, Putumayo, Casanare, Caquetá y Guaviare- En el momento de su fundación, la CNTV le autoriza como zona de influencia de su señal los departamentos 
de Cundinamarca, Boyacá, Tolima, Huila, Meta, Guaviare, Vaupés, Caquetá, Putumayo, Amazonas, Guainía, Vichada, Casanare y Arauca.

El Canal con sede en Bogotá, cuando en 1998 inicia sus emisiones, prometía ser una alternativa importante para que, productoras con experiencia en la televisión nacional formaran sus empresas de programación con buenos márgenes de ganancia, dado que ofrecía un cubrimiento de por lo menos 14 de departamentos, con tarifas regionales en la emisión y casi con un cubrimiento nacional para la comercialización.

Sin embargo esto no fue así con el correr de los meses. Las productoras que participaron en la licitación y ganaron la adjudicación de horarios, vieron defraudadas sus expectativas, pues recibieron entre otras ofertas del Canal, el compromiso de instalar repetidoras para transportar la señal a un vasto territorio y no fue así. Las disputas entre sus socios y sucesivos equívocos administrativos llevaron al Canal a la debacle por el incumplimiento de los compromisos. Dichos incumplimientos con los contratistas llevaron al retiro masivo de los mismos, y por consiguiente, a la obligación del Canal de asumir horas de programación propia, que no tenía presupuestado.

Esa condición de programador absoluto de su oferta, condujo a Tvandina a presentar una programación que no planteaba una propuesta para la región, pues a falta de ingresos por el alquiler de horarios y por la comercialización, nutrió su rejilla con producciones musicales y una que otra producción extranjera. Sobre transmisiones de interés para la región en sus primeros años fueron pocas las experiencias. Sobre la crisis de este Canal que nunca operó en sus inicios como un medio de expresión de los territorios que cubre, se refiere el periódico El Colombiano en su edición del 6 de abril de 2001, en la página $6 \mathrm{D}$ :

\footnotetext{
“...Directivos de este canal reunidos ayer en Junta Directiva comenzaron a trazar una estrategia para establecer de donde pueden provenir los recursos para salvarlo de su liquidación y cierre".
}

Los miembros de la Junta advirtieron la necesidad de un 'salvavidas' para que Tvandina solucione, en el corto o mediano plazo, la crisis de sus finanzas, y aunque no quedó definido si Telecom, la Comisión Nacional de Televisión, los departamentos o todos en conjunto aportarán recursos para llegar a una solución.

\subsubsection{Televisión Regional del Oriente,} TRO

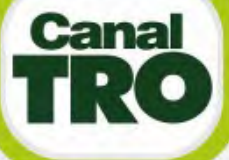

POR LA INTEERACIÓN
Este Canal constituido mediante escritura pública el 22 de junio de 1995, sirve con su señal a los departamentos de Santander y Norte de Santander, cuyas ciudades capitales son Bucaramanga y Cúcuta respectivamente. Para los habitantes de la región el Canal es el medio de comunicación visual de lo que se ha denominado el Gran Santander.

En acto público con presidente de la república incluido, ese 22 de junio firmaron la escritura de constitución como asociados: INRAVISIÓN, los departamentos de Norte de Santander y Santander, el Municipio de Bucaramanga, Infinorte, las Empresas Públicas de Bucaramanga y la Beneficencia de Santander.

Como muchos de los hechos propios de un país santanderista, de leyes, como lo es Colombia, el TRO que se constituyó con escritura pública ese 22 de junio de 1995, sólo después de 5 meses, recibió los aportes de capital, y sólo 4 años después, en 1999, ofreció su primera parrilla de programación propia, ya que durante los años anteriores realizó una señal de prueba, que aunque con alguna producción de la región, distaba mucho de ser una oferta de canal regional. Con la entrega de los aportes inicia entonces el proceso de configuración del Canal, se define que la sede será en la ciudad de Bucaramanga y se dan los primeros pasos para la adquisición de equipos de producción y transmisión.

El TRO, deberá afrontar durante sus primeros años dificultades acarreadas, no sólo por sus precario presupuesto sino por una indefinición en la asignación de frecuencias de transmisión por parte de la Comisión Nacional de Televisión, al modificarle en 1996, la asignada a la ciudad de Bucaramanga de VHF a UHF. Con un perjuicio enorme para su montaje técnico y con unas inversiones ya realizadas, después de muchas discusiones, la Comisión Nacional de Televisión, define por fin, que dicho canal operará para todos los dos departamentos en la frecuencia VHF.

Para el 2001, con una oferta de 11 horas de programación diaria, de las cuales el 50\% corresponden a producción propia, los pobladores de esta región estaban apenas estaban identificando su propia señal, pues su aparición como oferta de programación debió competir con el sinnúmero de señales satelitales, canales venezolanos por ser zona de frontera, 
de parabólicas y de los dos canales nacionales de operación privada, que en medio de una país en recesión, acapararon los mayores índices de sintonía y penetración.

\subsubsection{Teleislas}

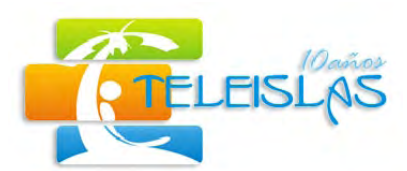

En 1974 cuando ni la televisión regional ni local estaban autorizadas en Colombia, en la isla de San Andrés se crea la estación local de televisión Simón Bolívar como una dependencia de INRAVISIÓN. La operación de esta estación podría llevar a afirmar que se constituyó en la primera experiencia de producción regional en Colombia, ya que se dota como un canal independiente pues tenía estudio y contaba con cámaras y equipos para la edición. Desde 1974 hasta 1991, casi en un ejercicio propio de un canal independiente, pues se autoriza incluso la emisión de patrocinios locales, los isleños produjeron su propia televisión, la cual programaron con la oferta de producción nacional recibida desde INRAVISIÓN.

En 1991, la estación autónoma de televisión deja de programarse independientemente y pasa a ser aparte de la red nacional. La estación entra a administrar el complejo sistema de microondas instalado por INRAVISIÓN para la Isla. Este sistema tenía como esquema transportar la señal desde Bogotá hasta la estación satelital de Chocontá que la transmite al satélite, señal que se recibe por la antena satelital de San Andrés ubicada en Telecom, la cual se transporta por microondas hasta la estación autónoma y de allí a los isleños.

Este complejo sistema de transporte de señal hacía que las fallas permanentes llevaran a sus administradores a emitir desde esa estación autónoma, actual sede de Teleislas, programación pregrabada nacional. Con ese nuevo esquema de transmisión se deja de producir programas propios.

Una vez Colombia incorpora la tecnología satelital en su sistema de transporte de señal, San Andrés y Providencia recibe en directo la televisión nacional, y en la región se inicia el trámite para crear su canal propio. Con una experiencia valiosa en la producción regional, Teleislas se constituyó en 1997 mediante escritura pública, como una sociedad entre INRAVISIÓN, el Departamento de San Andrés y el Municipio de Providencia. Este Canal origina para el 2001 su señal de prueba desde la estación autónoma, propiedad de INRAVISIÓN.
Sin embargo, pese a que legalmente en el 2001 estaba constituido el canal Teleislas, y que estaba generando señal de prueba con programas entregados por INRAVISIÓN y el Ministerio de Cultura, su funcionamiento como canal regional no fue posible en su totalidad, pues se presentó una disputa política entre sus socios, sucesivos Gobernadores de San Andrés y Alcaldes de Providencia, que imposibilitaron el funcionamiento.

Afirma el ingeniero Walt Hayes Bryan, empleado de INRAVISIÓN para la época y administrador de la estación autónoma por muchos años, que para el segundo semestre de 2001, resueltos los inconvenientes políticos, el canal regional entró a emitir su señal definitiva, ya que en abril del 2001 tanto la Alcaldía de Providencia, la Gobernación de San Andrés, la CNTV como INRAVISIÓN, autorizaron al Director de este último ente, a asumir como representante legal a fin de organizar la operación del mismo.

\section{Conclusiones}

Mientras en la mayoría de los países, que tienen estaciones propias de televisión en sus territorios, los canales de televisión son de dos dueños, el Estado por un lado, y los particulares por otro, en Colombia, el modelo fue diferente por 45 años. Dado su origen improvisado y como respuesta a una necesidad propagandística del dictador que la fundó, sin un capital que sustentara una oferta de programación de canal público educativo y cultural, como le correspondía al Estado que le dio nacimiento.

Con esta condición como origen, el Estado Colombiano ve en los particulares la posibilidad de financiar el sostenimiento de la naciente televisión, por eso autoriza en 1955, al año y dos meses de su creación, delegar a la empresa TVC, Televisión Comercial, una sociedad de las empresas radiales Caracol y Radio Cadena Nacional, RCN, la explotación comercial de algunos espacios en la programación del canal nacional.

Con esta autorización se implanta el modelo mixto de la televisión colombiana, en el cual el Estado dueño de las frecuencias, arrienda a unos particulares espacios de televisión para que la exploten comercialmente, y de paso, la inversión en la televisión que le correspondía hacer, la educativa y cultural, la financiaba para presentarla en los espacios de menor audiencia.

Durante 45 años, la televisión educativa y cultural presentada en los horarios cola, y en el canal cultural fundado en la década de los $60 \mathrm{y}$ autorizado para tener cubrimiento nacional en 1985, no ha tenido una política sistemática en la programación y en la financiación. 
En un contexto político como el que vivió Colombia en la segunda mitad del siglo XX, se entiende también la necesidad del Estado a través de sus distintos gobiernos, de controlar la televisión con el mantenimiento del modelo mixto.

Para concluir, se puede decir que en el modelo colombiano convivieron de un lado, unos particulares que se enriquecieron y utilizaron la televisión con fines eminentemente comerciales, y por otro, un Estado que abandonó su función educadora, en un medio que exigía su compromiso.

Al iniciar la década de los 80, las demandas para que se crearan canales regionales crecieron, los antioqueños, costeños y vallecaucanos, fueron quienes más clamaron por la creación de canales de televisión que estuvieran cerca del sentir regional.

TELEANTIOQUIA se crea el 11 de agosto de 1985, ocho meses después de firmados los Decretos Nacionales 3100 y 3101en 1984, durante la presidencia del antioqueño, Belisario Betancur Cuartas. Telecaribe surge en abril de 1986, Telepacífico el 8 julio de 1988, Telecafé surge el 17 de octubre de 1992, Canal Capital en 1995, Tvandina - Canal 13 en la actualidad se constituye en 1995, Televisión Regional del Oriente -TROse crea el 22 de junio de 1995 y Teleislas se constituyó en 1997.

\section{Bibliografía}

Alzate Ritcher, Angela María, Berrío Solarte Hubert y Joaquín Restrepo Mejía. LAS TELECOMUNICACIONES EN ANTIOQUIA. UN SIGLO DE HISTORIA. Editorial Universidad Pontificia Bolivariana, Medellín, 1999, 169 pgs.

Abello Banfi, Jaime. LA TELEVISIÓN REGIONAL. Edición Dominical del Periódico El Heraldo, Barranquilla, 23 de octubre de 1994.

COLOMBIA PAÍS DE REGIONES. Suplemento Educativo, periódico El Colombiano 1994, 592 pgs.

COLOMBIA VIVA. Suplemento Educativo, periódico El Tiempo, 2000, 300 pgs.

CONSTITUCIÓN POLÍTICA DE COLOMBIA. Editorial Temis, Santafé de Bogotá, vigesimaprimera edición, 1991, 231 pgs.

DEL MAGUARÉ A LA FIBRA ÓPTICA. CRÓNICA DE LAS COMUNICACIONES.

Publicación del Ministerio de Comunicaciones de Colombia y TELECOM, Bogotá 1995, 369 pgs.
García Canclini, Néstor. CONSUMIDORES Y CIUDADANOS. CONFLICTOSMULTICULTURALES DE LA GLOBALIZACIÓN. Editorial Grijalbo, México D.F., 1995, 198 pgs.

GeaRifà,MiguelÁngel.TELEVISIÓNREGIONAL Y DESARROLLO. APROXIMACIÓN A LA EXPERIENCIA COLOMBIANA. Aconcagua Libros. Unidad de Gestión de La Rábida. Diputación de Huelva. Colección La Otra América, Huelva, 1999, 186 pgs

HACIA UN NUEVO CONCEPTO DE TELEVISIÓN. Libro publicado por RTVV.

Valencia, España, junio de 1998. 252 pgs. Libro publicado por RTVV. Valencia, España, junio de 1997. 262 pgs.

HISTORIA DE UNA TRAVESÍA. CUARENTA AÑOS DE LA TELEVISIÓN EN COLOMBIA. Publicación del Instituto Nacional de Radio y Televisión, INRAVISION, julio de 1994, 520 pgs.

\section{Notas}

[1] Historia de una travesía. Cuarenta años de la televisión en Colombia. Publicación del Instituto Nacional de Radio y Televisión, INRAVISION, julio de 1994. 520 pgs. p.p.35.

[2] Idem. p.p. 67

\section{Para citar este artículo:}

García, Ana Patricia (2012). TELEVISIÓN EN COLOMBIA: Surgimiento de los canales regionales. Revista Virtual Luciérnaga, Año 4, N7. Grupo de Investigación en Comunicación, Facultad de Comunicación Audiovisual, Politécnico Colombiano Jaime Isaza Cadavid. MedellínColombia. ISSN 2017-1557. Págs. 23-35.

DOI. 10.33571/revistaluciernaga.v4n7a3 